
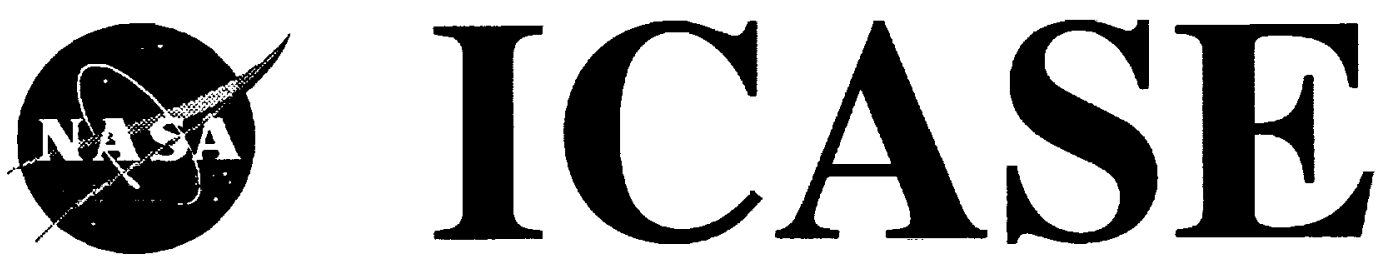

\title{
A NUMERICAL METHOD FOR SOLVING THE THREE-DIMENSIONAL PARABOLIZED NAVIER- STOKES EQUATIONS
}

Domenic D'Ambrosio Roberto Marsilio

(NASA-CR-198159) A NUMERICAL METHOD FOR SOLVING THE

THREE-DIMENSIONAL PARABOLIZED NAVIER-STCKES EQUATIONS FinaI

Contract No. NAS1-19480

May 1995

Institute for Computer Applications in Science and Engineering

NASA Langley Research Center

Hampton, VA 23681-000I 


\title{
A Numerical Method for Solving the Three-Dimensional Parabolized Navier-Stokes Equations *
}

\author{
Domenic D'Ambrosio \\ Dipartimento di Ingegneria Aeronautica e Spaziale \\ Politecnico di Torino \\ 10129 Torino, Italy \\ Roberto Marsilio \\ Institute for Computer Applications in Science and Engineering \\ NASA Langley Research Center \\ Hampton, VA 23681, USA.
}

\begin{abstract}
A numerical technique that solves the parabolized form of the Navier-Stokes equations is presented. Such a method makes it possible to obtain very detailed descriptions of the flowfield in a relatively modest CPU time. The present approach is based on a space-marching technique, uses a finite volume discretization and an upwind fluxdifference splitting scheme for the evaluation of the inviscid fluxes. Second order accuracy is achieved following the guidelines of the the ENO schemes. The methodology is used to investigate three-dimensional supersonic viscous flows over symmetric corners. Primary and secondary streamwise vortical structures embedded in the boundary layer and originated by the interaction with shock waves are detected and studied. For purpose of validation, results are compared with experimental data extracted from literature. The agreement is found to be satisfactory. In conclusion, the numerical method proposed seems to be promising as it permits, at a reasonable computational expense, investigation of complex three-dimensional flowfields in great detail.
\end{abstract}

*This research was supported in part by MPI40\% and it was also supported in part by the National Aeronautics and Space Administration under NASA Contract No. NAS1-19480 while the second author was in residence at the Institute for Computer Applications in Science and Engineering, (ICASE), NASA Langley Research Center, Hampton, VA, 23681 


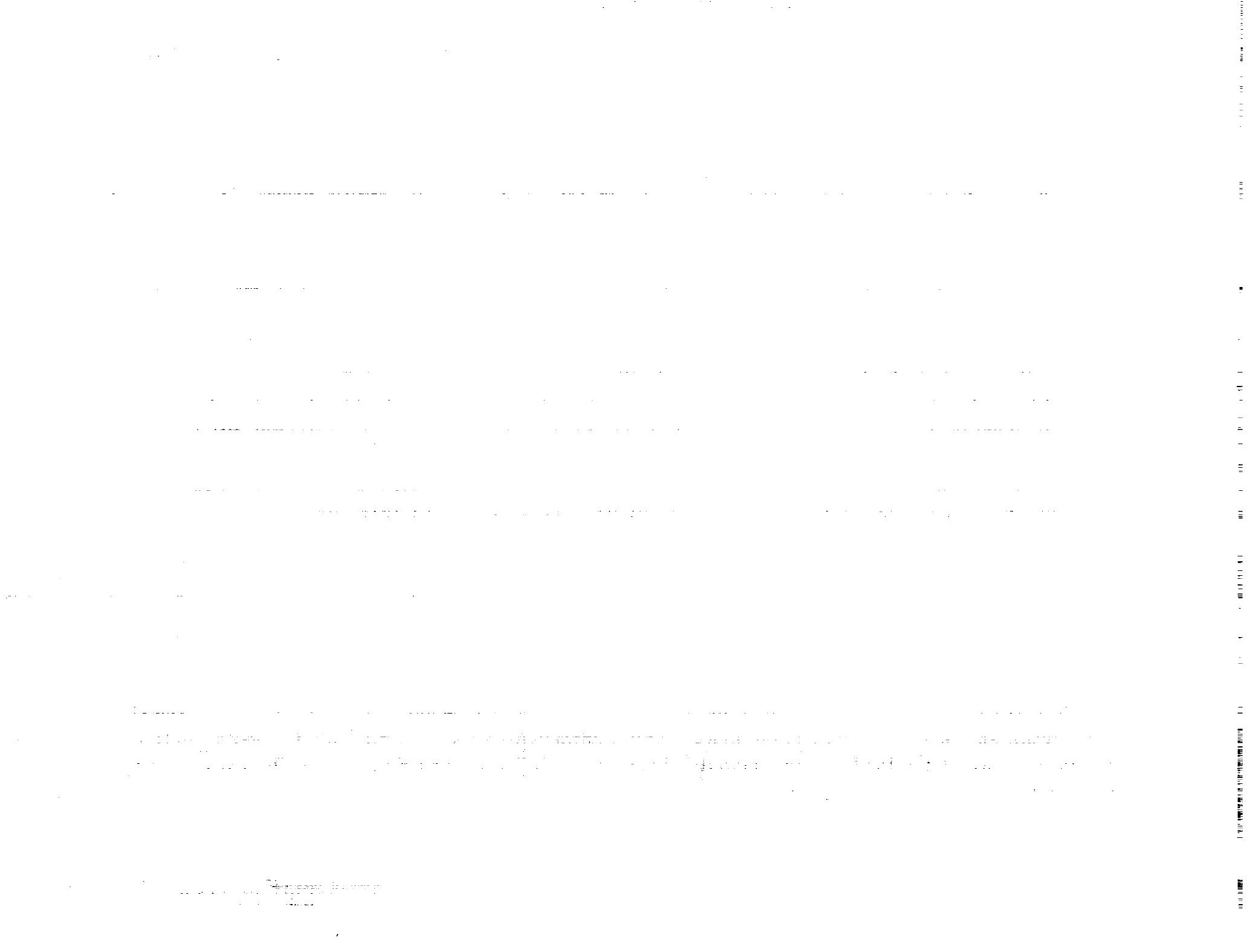




\section{Introduction}

In the study of three-dimensional supersonic flows it is common to be faced with complex interactions concerning shock waves and viscous layers. Such occurrences often provoke dramatic changes in the flowfield features, both qualitatively and quantitatively. Shockinduced separations of the viscous layer make the flow vortex-dominated close to the wall, and vortical structures are also likely to appear, for sufficiently high Reynolds numbers, in zones where convective effects are preponderant, due the instability of the slip surfaces resulting from shock/shock interactions. Important consequences of these interactions are increases in heat fluxes, skin friction coefficients and pressures at the wall in correspondence with the reattachment of the separated flow; in addition transition, shock wave shapes and the efficiency of the air-intakes that might swallow such streams are affected.

Numerical tests are usually helpful in the investigations of such complicated fluid-dynamic patterns, as they provide a tool to observe and possibly to understand the origins and the effects of the numerous phenomena triggered by shock/shock and shock/viscous layer interactions. It is clear, however, that in order to obtain good numerical results comparable with experimental data, a fairly detailed description of the flowfield is necessary, especially when multiple vortical structures are present.

The only completely correct way of solving numerically three-dimensional compressible viscous flows is to integrate in time the full Navier-Stokes equations until a steady-state (if one exists) is reached. This approach is certainly affordable today, but, if many grid points are needed to solve in detail complex fluid-dynamic features, it could be excessively time and memory consuming. In the case of supersonic steady-state flows, however, this practical difficulty can be partially circumvented with the aid of the approximate form of the full Navier-Stokes equations known as Parabolized Navier-Stokes equations.

As will be pointed out in the next sections, the advantage of the Parabolized NavierStokes (PNS) equations is that they can be solved using a space-marching technique, a characteristic which allows one to spend relatively short computational effort and also results in noticeable memory savings. Therefore, it is possible to reinvest time and memory in more refined grids, thus permitting a better resolution of the flowfield. As a drawback, the parabolizing assumption requires the freestream Mach number to be supersonic and the streamwise velocity to be always positive (streamwise flow separations are thus excluded, while crossflow separations are permitted); moreover, the streamwise pressure gradient must be altered in the subsonic part of the flowfield [1].

In the approach presented here, the governing equations are integrated in an explicit fashion and the physical domain is discretized according to a finite volume technique. The convective part of the equations (inviscid fluxes) is treated following a flux-difference-splitting method with an approximate solution of a Riemann problem at each cell interface [2][3] while the diffusive terms (viscous fluxes) are calculated using a centered scheme. Second order accuracy is achieved by means of an Essentially Non Oscillatory scheme [4] with linear reconstruction of the solution at each step of integration. Presently, only inert gases in laminar regime are considered, but a future extension to include thermochemical or turbulence effects is certainly possible.

To validate the method, numerical results are compared with experimental data extracted from the literature. Supersonic corner flows configurations have been chosen as a 
benchmark as they give the chance to have, on the same geometry, shock/shock interactions and shock/viscous layer interactions with multiple separations. The comparison appears to be satisfactory, as the same flowfield features are recognized, and measured and computed values show a good agreement.

\section{Governing equations}

\subsection{Starting point: the three-dimensional Navier-Stokes equations}

Compressible viscous flows are governed by the Navier-Stokes equations, that written in integral conservative form read like:

$$
\frac{\partial}{\partial t} \int_{\mathcal{V}} \mathbf{W} d \mathcal{V}+\int_{\mathcal{S}} \mathbf{F}_{\mathbf{I}} \cdot \vec{n} d \mathcal{S}+\int_{\mathcal{S}} \mathbf{F}_{\mathbf{V}} \cdot \vec{n} d \mathcal{S}=0
$$

where $\mathcal{V}$ represents an arbitrary volume inclosed in a surface $\mathcal{S}$ with unit normal $\vec{n}$ positive if directed outward.

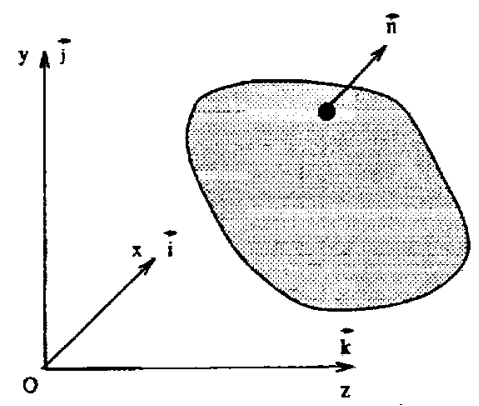

Figure 1: Control volume

System (1) can be reduced to the non-dimensional form with the help of the following reference values: $L$ for length, $T_{\infty}$ for temperature, $\sqrt{R T_{\infty}}$ for velocity, $R T_{\infty}$ for energy per unit mass and $\mu\left(T_{\infty}\right)$ for viscosity. Therefore, from now on, the flowfield variables should be considered as non-dimensional. In particular, $\mathbf{W}$ is the vector of conservative variables

$$
\mathbf{W}=\{\rho, \rho \vec{q}, E\}^{T}
$$

tensor $\mathbf{F}_{\mathbf{I}}$ contains the inviscid fluxes

$$
\mathbf{F}_{\mathbf{I}}=\{\rho \vec{q}, p \bar{I}+\rho \vec{q} \otimes \vec{q},(E+p) \vec{q}\}^{T}
$$

and tensor $\mathbf{F} \mathbf{v}$ contains the viscous fluxes

$$
\mathbf{F}_{\mathbf{V}}=\frac{\sqrt{\gamma} M_{\infty}}{R e_{\infty}}\{\overrightarrow{0},-\overline{\bar{\tau}},-k \nabla T-\overline{\bar{\tau}} \cdot \vec{q}\}^{T}
$$

Quantities $\rho, p$ and $\vec{q}=\{u, v, w\}^{T}$ are respectively the local density, pressure and velocity; $E$ represents the total energy per unit volume:

$$
E=\rho\left(e+\frac{|\vec{q}|^{2}}{2}\right)
$$


$e$ is the internal energy per unit mass, $M_{\infty}$ and $R e_{\infty}$ are the freestream Mach number and Reynolds number, $\gamma$ is the ratio of the specific heats and, finally, $\bar{I}$ is the unit matrix.

Viscous stresses are contained in tensor $\overline{\bar{\tau}}$, with

$$
\tau_{i j}=\mu\left[\left(\frac{\partial q_{j}}{\partial x_{i}}+\frac{\partial q_{i}}{\partial x_{j}}\right)-\frac{2}{3}(\nabla \vec{q}) \delta_{i j}\right]
$$

where $\delta_{i j}$ is the Kröneker's symbol. The viscosity is calculated via Sutherland's law:

$$
\mu=T^{3 / 2}\left(\frac{1+T_{\text {ref }}}{T+T_{\text {ref }}}\right)
$$

where

$$
T_{\text {ref }}=\frac{110.4 K}{T_{\infty}}
$$

and the thermal conductivity $k$ is obtained according to the relation:

$$
k=\frac{\mu}{\operatorname{Pr}} \frac{\gamma}{\gamma-1}
$$

where $\operatorname{Pr}$ is the Prandtl number. Finally, the perfect gas relationship completes the set of equations

$$
\frac{p}{\rho}=T
$$

\subsection{Three-dimensional Parabolized Navier-Stokes equations}

The three-dimensional Parabolized Navier-Stokes equations are derived from the steady-state full Navier-Stokes equations with the aim of obtaining a system of equations representing a well posed problem with respect to an integration performed using a space-marching technique. For this purpose, any derivative in the streamwise direction contained in the stress tensor is neglected, all viscous and heat fluxes in the streamwise direction are dropped and the pressure gradient in the subsonic layer is properly altered. With such modifications, which are valid only for sufficiently high Reynolds numbers, system (1) is reduced to a set of hyperbolic-parabolic equations [1][5].

Thus, assuming the streamwise direction to be coincident with the $\mathrm{x}$-axis, the components of the stress tensor necessary to evaluate the viscous fluxes are reduced to the following form:

$$
\begin{aligned}
\tau_{x x}^{*} & =-\frac{2}{3} \mu\left(v_{y}+w_{z}\right) \\
\tau_{y y}^{*} & =\frac{2}{3} \mu\left(2 v_{y}-w_{z}\right) \\
\tau_{z z}^{*} & =\frac{2}{3} \mu\left(2 w_{z}-v_{y}\right) \\
\tau_{x y}^{*} & =\tau_{y x}^{*}=\mu u_{y} \\
\tau_{x z}^{*} & =\tau_{z x}^{*}=\mu u_{z} \\
\tau_{y z}^{*} & =\tau_{z y}^{*}=\mu\left(v_{z}+w_{y}\right)
\end{aligned}
$$


Moreover, the $\mathrm{x}$-component of the temperature gradient is neglected:

$$
\nabla T^{*}=T_{y} \vec{j}+T_{z} \vec{k}
$$

The streamwise pressure gradient is split according to the technique suggested by Vigneron, Rakich and Tannehill in reference [1]:

$$
p_{x}=\omega p_{x}+(1-\omega) p_{x}
$$

If only the first term of the RHS of equation (15) is retained and the second term is considered as a source term, the set of equations containing only the convective terms is hyperbolic provided that

$$
\left\{\begin{array}{l}
u>0 \\
\omega<\frac{\gamma M_{x}^{2}}{1+(\gamma-1) M_{x}^{2}} \quad \text { for } \quad M_{x}<1
\end{array}\right.
$$

where $M_{x}$ is the local Mach number in the streamwise direction. The first condition contained in equations (16) prevents the PNS approximation from being used when the flow separates in the streamwise direction. A short analysis of the second condition reveals that $\omega$ is equal to 1 when $M_{x}=1$ and is zero when $M_{x}=0$; this means that the effect of the streamwise pressure gradient is completely neglected at the wall, but is progressively included as the flow approaches supersonic conditions. For $M_{x}$ greater than 1 the effect of $p_{x}$ will be entirely taken into account and the value of $\omega$ will be unity. Of course, since a space-marching integration is desired, the freestream Mach number will necessarily have to be supersonic.

Combining the cited assumptions, system (1) is finally reduced to the following form:

$$
\int_{\mathcal{S}} \mathbf{F}_{\mathbf{I}}^{*} \cdot \vec{n} d \mathcal{S}+\int_{\mathcal{S}} \mathbf{F}_{\mathbf{V}}^{*} \cdot \overrightarrow{n^{*}} d \mathcal{S}=\int_{\mathcal{S}} \mathbf{P} \cdot \vec{n} d \mathcal{S}+\mathbf{P}^{\prime}
$$

where

$$
\begin{aligned}
& \mathbf{F}_{\mathrm{I}}^{*}=\left\{\rho \vec{q}, p \overline{I^{*}}+\rho \vec{q} \otimes \vec{q},(E+p) \vec{q}\right\}^{T} \\
& \mathbf{F}_{\mathbf{V}}^{*}=\frac{\sqrt{\gamma} M_{\infty}}{R e_{\infty}}\left\{\overrightarrow{0},-\overline{\overline{\tau^{*}}},-k \nabla T^{*}-\overline{\overline{\tau^{*}}} \cdot \vec{q}\right\}^{T} \\
& \mathbf{P}=\left\{\overrightarrow{0},-(1-\omega) p \overline{I^{* *}}, \overrightarrow{0}\right\}^{T}
\end{aligned}
$$

and $\overline{I^{*}}$ and $\overline{I^{* *}}$ are scalar matrices with:

$$
\begin{aligned}
& \operatorname{diag} \overline{I^{*}}=(\omega, 1,1) \\
& \operatorname{diag} \overline{I^{* *}}=(1,0,0)
\end{aligned}
$$

Since the viscous and heat fluxes in the steamwise direction are dropped, vector $\overrightarrow{n^{*}}$ contains only the components in the $y$-direction and in the $z$-direction of the normal unit vector, that is $\overrightarrow{n^{*}}=\left(0, n_{y}, n_{z}\right)$.

A source term $\mathbf{P}^{\prime}$ must be added to make the integral formulation coherent with equation (15), as noticed in references [6] and [7]. In fact, the corresponding integral form of $\omega p_{x}$ is:

$$
\omega \int p n_{x} d \mathcal{S}=\int \omega p n_{x} d \mathcal{S}-p \int \omega n_{x} d \mathcal{S}
$$




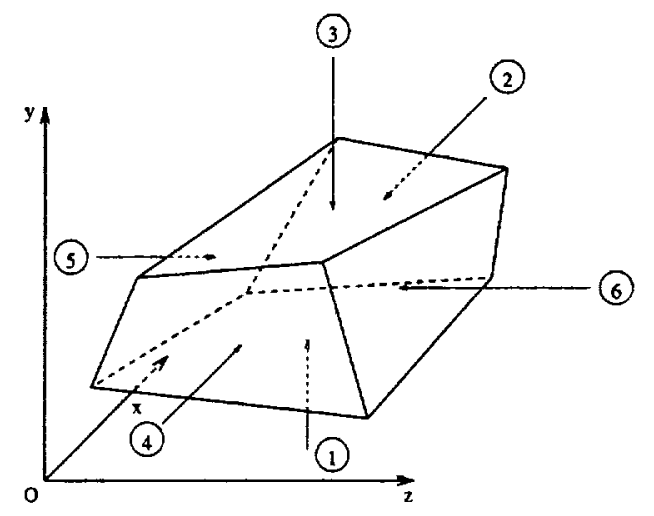

Figure 2: Computational control volume

Therefore, we have:

$$
\mathbf{P}^{\prime}=\{\overrightarrow{0}, \bar{\Omega}, \overrightarrow{0}\}^{T}
$$

where $\bar{\Omega}$ is a scalar matrix with

$$
\operatorname{diag} \bar{\Omega}=\left(p \int \omega n_{x} d \mathcal{S}, 0,0\right)
$$

Real gas effects, that can arise for Mach numbers above 6, are neglected here. Moreover, the flowfield regime is assumed to be laminar.

\section{Numerical solution of the PNS equations}

\subsection{Discretization and integration}

Being a set of hyperbolic-parabolic equations, PNS equations can be integrated in the streamwise direction according to a space-marching technique. In the present approach, such a direction is assumed to be coincident with the $\mathrm{x}$-axis, so that the flowfield is solved step by step in planes normal to the $\mathrm{x}$-direction. The physical domain is discretized according to a finite volume approximation. Computational control volumes, as shown in figure 2 , are hexahedrons having two faces (labeled 2 and 4) normal to the $x$-direction. Keeping in mind the previous discussion, system (17) can be discretized in the following way:

$$
\sum_{i=1}^{6}\left(\mathbf{F}_{\mathbf{I}}^{*}\right)_{i} \cdot \vec{n}_{i} \Delta \mathcal{S}_{i}+\sum_{i=1}^{6}\left(\mathbf{F}_{\mathbf{V}}^{*}\right)_{i} \cdot \vec{n}_{i}{ }_{i} \Delta \mathcal{S}_{i}-\sum_{i=1}^{6}\left(\mathbf{P}^{\prime}\right)_{i}=0
$$

where $i$ is the surface label.

In equation (22), $\left(\mathbf{F}_{\mathbb{I}}^{*}\right)_{4}$ is the solution obtained at the previous integration step and $\left[\left(\mathbf{F}_{\mathbf{I}}^{*}\right)_{2}-\left(\mathbf{P}^{\prime}\right)_{2}\right]$ is the unknown. The remaining inviscid and viscous fluxes across the lateral surfaces have to be estimated and this will be the subject of the next two subsections. The source term $\mathbf{P}$ has been omitted in equation (22), this implies that only a part of the streamwise pressure gradient is considered in the subsonic layer. The reason for neglecting $\mathbf{P}$ is that it should be evaluated using a backward difference (i.e. considering the previous 
volume) and this, as explained in reference [1], would arise in a stability condition imposing a lower bound for $\Delta x$.

The integration of equation (22) is performed in an explicit manner, so that the values of the primitives variables that are used to evaluate the lateral fluxes are those that have been already calculated at the previous step, that is on surface 4 . The x-momentum component of $\mathbf{P}^{\prime}$, which is the only one different from zero (see equations (20) and (21)), is discretized as:

$$
\begin{aligned}
P_{2}^{\prime} & =p_{4}\left(\omega_{4} \vec{n}_{x_{4}} \Delta \mathcal{S}_{4}+\omega_{1} \vec{n}_{x_{1}} \Delta \mathcal{S}_{1}+\omega_{3} \vec{n}_{x_{3}} \Delta \mathcal{S}_{3}+\omega_{5} \vec{n}_{x_{5}} \Delta \mathcal{S}_{5}+\omega_{6} \vec{n}_{x_{6}} \Delta \mathcal{S}_{6}+\omega_{2} \vec{n}_{x_{2}} \Delta \mathcal{S}_{2}\right)= \\
& =\left(\mathbf{P}^{\prime}\right)_{4}+\left(\mathbf{P}^{\prime}\right)_{1}+\left(\mathbf{P}^{\prime}\right)_{3}+\left(\mathbf{P}^{\prime}\right)_{5}+\left(\mathbf{P}^{\prime}\right)_{6}+\left(\mathbf{P}^{\prime}\right)_{2}
\end{aligned}
$$

The unknown fluxes $\left[\left(\mathbf{F}_{\mathrm{I}}^{*}\right)_{2}-\left(\mathbf{P}^{\prime}\right)_{2}\right]$ are finally evaluated from equation (22):

$$
\begin{aligned}
{\left[\left(\mathbf{F}_{\mathrm{I}}^{*}\right)_{2}-\left(\mathbf{P}^{\prime}\right)_{2}\right] \cdot \vec{n}_{2} \Delta \mathcal{S}_{2}=- } & {\left[\left(\mathbf{F}_{\mathrm{I}}^{*}\right)_{4} \cdot \vec{n}_{4} \Delta \mathcal{S}_{4}-\left(\mathbf{P}^{\prime}\right)_{4}+\right.} \\
& \left(\mathbf{F}_{\mathrm{I}}^{*}\right)_{1} \cdot \vec{n}_{1} \Delta \mathcal{S}_{1}+\left(\mathbf{F}_{\mathbf{V}}^{*}\right)_{1} \cdot \vec{n}^{*}{ }_{1} \Delta \mathcal{S}_{1}-\left(\mathbf{P}^{\prime}\right)_{1}+ \\
& \left(\mathbf{F}_{\mathrm{I}}^{*}\right)_{3} \cdot \vec{n}_{3} \Delta \mathcal{S}_{3}+\left(\mathbf{F}_{\mathbf{V}}^{*}\right)_{3} \cdot \vec{n}^{*}{ }_{3} \Delta \mathcal{S}_{3}-\left(\mathbf{P}^{\prime}\right)_{3}+ \\
& \left(\mathbf{F}_{\mathrm{I}}^{*}\right)_{5} \cdot \vec{n}_{5} \Delta \mathcal{S}_{5}+\left(\mathbf{F}_{\mathrm{V}}^{*}\right)_{5} \cdot \vec{n}^{*}{ }_{5} \Delta \mathcal{S}_{5}-\left(\mathbf{P}^{\prime}\right)_{5}+ \\
& \left.\left(\mathbf{F}_{\mathrm{I}}^{*}\right)_{6} \cdot \vec{n}_{6} \Delta \mathcal{S}_{6}+\left(\mathbf{F}_{\mathbf{V}}^{*}\right)_{6} \cdot \vec{n}^{*}{ }_{6} \Delta \mathcal{S}_{6}-\left(\mathbf{P}^{\prime}\right)_{6}\right]
\end{aligned}
$$

To terminate the step, it is necessary to extract the primitive variables from the solution fluxes $\left[\left(\mathbf{F}_{\mathbf{I}}^{*}\right)_{2}-\left(\mathbf{P}^{\prime}\right)_{2}\right]$, which contain $\omega$. To accomplish this, a cubic function $\omega=\omega\left(\mathbf{F}_{\mathbf{I}}^{*}-\mathbf{P}^{\prime}\right)$ has been obtained starting from the cubic function $\omega=\omega\left(\mathbf{F}_{\mathbf{I}}^{*}\right)$ suggested by J. Korte in [6]. Denoting the $j$ th component of $\left[\left(\mathbf{F}_{\mathbf{I}}^{*}\right)_{2}-\left(\mathbf{P}^{\prime}\right)_{2}\right]$ for brevity by $\left(F_{j}\right)_{s}$, the form of the function is:

$$
\begin{array}{r}
\left(1+B p_{4}\right) \omega_{2}^{3}-\left[\frac{2(1+\varsigma) \gamma}{\gamma-1}+2 B\left(\frac{\gamma \varsigma p_{4}}{\gamma-1}-\left(F_{2}\right)_{s}\right)\right] \omega_{2}^{2}+ \\
\quad\left[\frac{(1+\varsigma)^{2} \gamma^{2}+A}{(\gamma-1)^{2}}-4 \frac{\gamma}{\gamma-1} B \varsigma\left(F_{2}\right)_{s}\right] \omega_{2}-\frac{2 A \varsigma \gamma}{(\gamma-1)^{3}}=0
\end{array}
$$

with

$$
\begin{gathered}
A=\frac{\gamma^{2}\left(F_{2}\right)_{s}^{2}}{2\left(F_{5}\right)_{s}\left(F_{1}\right)_{s}-\left(F_{3}\right)_{s}^{2}-\left(F_{4}\right)_{s}^{2}} \\
B=\frac{\gamma^{2} p_{4}}{(\gamma-1)^{2}\left[2\left(F_{5}\right)_{s}\left(F_{1}\right)_{s}-\left(F_{3}\right)_{s}^{2}-\left(F_{4}\right)_{s}^{2}\right]}
\end{gathered}
$$

and where $p_{4}$ is the pressure on surface 4 . The quantity $\varsigma$ is a safety factor $(\sim 0.8)$ which is applied to $\omega$, that is evaluated at first according to equation (16), but is then corrected as:

$$
\omega=\min (1, \varsigma \omega)
$$

Equation (24) is solved using a Newton-Raphson iteration. Once $\omega_{2}$ is known, it is possible to evaluate $F_{I 2}^{*}$ and finally the primitive variables on the face labeled with 2 . 


\subsection{Evaluation of the lateral convective fluxes}

Lateral inviscid fluxes are evaluated defining and solving an appropriate Riemann problem across each lateral surface $(1,3,5$ or 6$)$.

The definition of the Riemann problem consists, at first, in considering the two finite volumes connected by the lateral surface and in fixing a direction $\eta$ joining the volumes and belonging to the $y-z$ plane that contains surfaces labeled as 4 . In the present approach $\eta$ is normal to the trace of the considered surface in the $y-z$ plane (see figure 3 ). Then, the variation of the flowfield variables along $\eta$ is to be considered. Due to the discretization, two piecewise constant (first order accuracy) or piecewise linear (second order accuracy) distributions of the flowfield variables are present between cells A and B, separated by a discontinuity in correspondence of the lateral surface (see figures 3 and 4). The collapse of such a discontinuity generates a pattern of waves along which signals propagate. The waves split the domain in the vicinity of the discontinuity in a set of uniform regions where the values of the flowfield variables are to be found, generating in this way a Riemann problem.

To obtain waves directions and corresponding signals, the equations governing the inviscid part of the flowfield are written in quasi-linear form in a new local frame of reference constituted by direction $\eta$, by the $\mathrm{x}$-direction and by a $\zeta$-direction normal to plane $\eta-x$ (see figure 3). Here an approximate solution of the Riemann problem is sought for [2], and consequently the Euler equations are written under the assumption of isentropic flow. Moreover, the Riemann problem is solved for simplicity in two dimensions rather than in three, so that only variations of the flowfield variables along the $\mathrm{x}-\eta$ plane are considered. Therefore, all the derivatives in the $\zeta$-direction are neglected, and finally the following set of equations is obtained:

$$
\left\{\begin{array}{l}
\kappa P_{x}+\tilde{\sigma} P_{\eta}+\gamma \tilde{\sigma}_{\eta}=0 \\
\tilde{\sigma}_{x}+\tilde{\sigma} \tilde{\sigma}_{\eta}+\frac{a^{2}}{\gamma u^{2}}\left(P_{\eta}-\tilde{\sigma} \omega P_{x}\right)=0 \\
\tilde{w}_{x}+\tilde{\sigma} \tilde{w}_{\eta}=0 \\
h_{x}+\tilde{\sigma} h_{\eta}-\frac{a^{2}}{\gamma}\left(\omega P_{x}+\tilde{\sigma} P_{\eta}\right)=0 \\
h_{x}^{o}+\tilde{\sigma} h_{\eta}^{o}=0
\end{array}\right.
$$

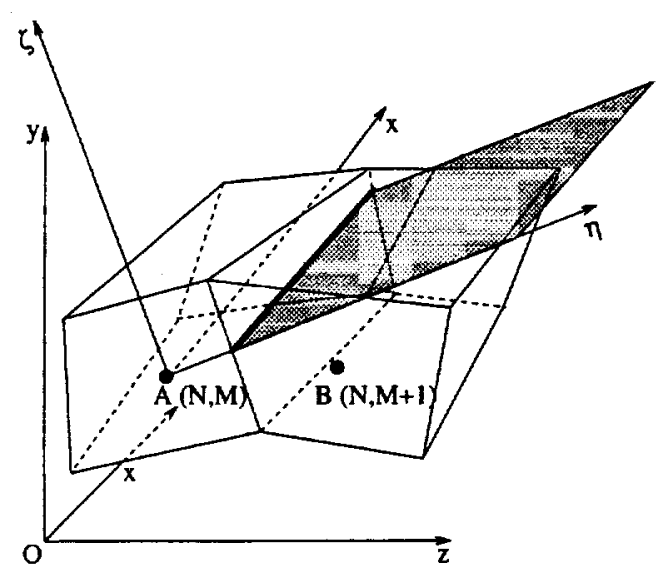

Figure 3: Local frame of reference 


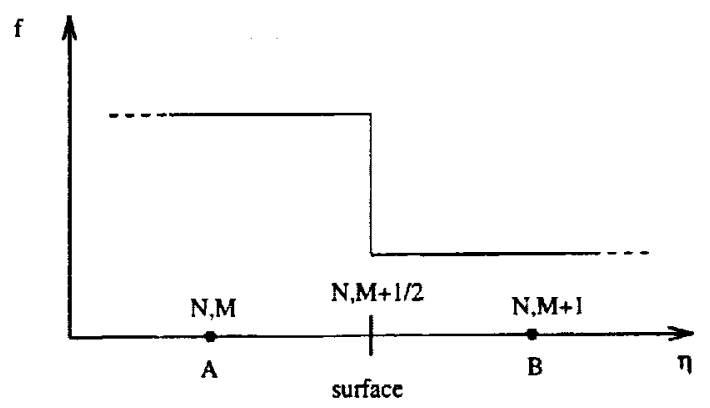

Figure 4: Piecewise constant distribution along $\eta$ between cells A and B

where $P, \tilde{\sigma}, \tilde{w}, h$, and $h^{\circ}$ have been chosen as dependent variables, with:

$$
\begin{aligned}
& \kappa=\gamma(1-\omega)+\omega\left(1-\frac{a^{2}}{u^{2}}\right) \\
& P=\ln p \\
& \tilde{\sigma}=\eta_{y} \sigma+\eta_{z} \tau \\
& \tilde{w}=\zeta_{y} v+\zeta_{z} w \\
& h^{\circ}=h+\frac{u^{2}+v^{2}+w^{2}}{2} \\
& \sigma=\frac{v}{u} \quad \tau=\frac{w}{u}
\end{aligned}
$$

and where $\vec{\eta}=\left(\eta_{y}, \eta_{z}\right)$ and $\vec{\zeta}=\left(\zeta_{y}, \zeta_{z}\right)$ are unit vectors defining directions $\eta$ and $\zeta$.

Owing to their hyperbolic nature, the quasilinear equations can be replaced by the compatibility equations that describe the convection of signals. In the present case, the collapse of the discontinuity between $A$ and $B$ generates a pattern of five waves (characteristic lines) along which the signals (Riemann invariants) defined by the compatibility equations propagate. The Riemann invariants and characteristic slopes corresponding to system (25) are:

$$
\left\{\begin{array}{l}
d R_{1,5}=d P+\frac{\gamma u^{2}}{\mp a^{2} \beta-u^{2} \tilde{\sigma}(\alpha-1)} d \tilde{\sigma}=0 \\
d R_{2}=d h-\frac{a^{2} \omega}{\gamma} \frac{(1+\tilde{\sigma})}{1+\omega \tilde{\sigma}^{2}} d P+\frac{u^{2} \tilde{\sigma}(1-\omega)}{1+\omega \tilde{\sigma}^{2}} d \tilde{\sigma}=0 \\
d R_{3}=d w=0 \\
d R_{4}=d h^{\circ}=0
\end{array}\right.
$$

and

$$
\left\{\begin{array}{l}
\lambda_{1,5}=\frac{\alpha u^{2} \tilde{\sigma} \mp a^{2} \beta}{\omega\left(u^{2}-a^{2}\right)+\gamma u^{2}(1-\omega)} \\
\lambda_{2,3,4}=\tilde{\sigma}
\end{array}\right.
$$

with

$$
\begin{aligned}
& \beta=\sqrt{\frac{u^{2}}{a^{2}}\left[\omega\left(1+\tilde{\sigma}^{2}\right)-\gamma(\omega-1)\right]-\omega+\left(\frac{u^{2}}{a^{2}}\right)^{2}\left(\frac{\gamma-1}{2}\right)^{2}(\omega-1)^{2} \tilde{\sigma}^{2}} \\
& \alpha=\frac{1}{2}[1+\omega+\gamma(1-\omega)]
\end{aligned}
$$




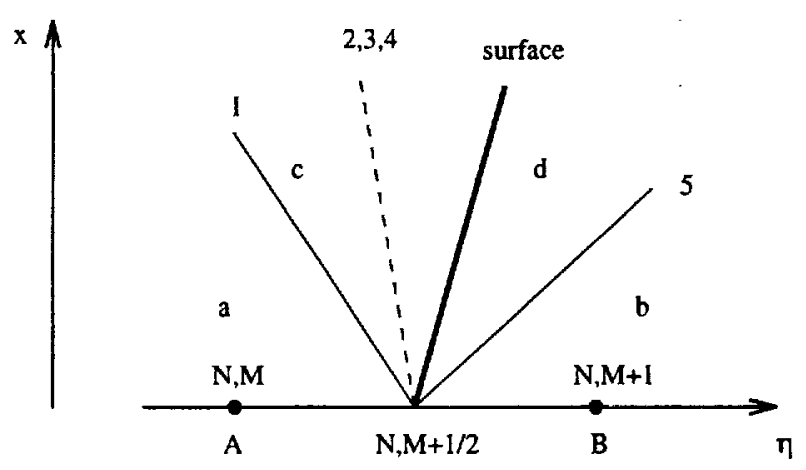

Figure 5: The Riemann problem

Equation (27) shows that there are only three distinct waves, which in the following will be called I (wave \#1), II (waves \#2,3,4) and III (wave \#5). Such waves generate two new uniform regions $c$ and $d$, which add up to the initial ones $a$ and $b$ (see figure 5). As signals traveling on each wave are not altered when crossing a characteristic of a different family, it is possible to write:

$$
\begin{array}{ll}
d R_{1_{b}}=d R_{1_{d}} & \\
d R_{2_{a}}=d R_{2_{c}} & d R_{2_{b}}=d R_{2_{d}} \\
d R_{3_{a}}=d R_{3_{c}} & d R_{3_{b}}=d R_{3_{d}} \\
d R_{4_{a}}=d R_{4_{c}} & d R_{4_{b}}=d R_{4_{d}} \\
d R_{5_{a}}=d R_{5_{c}} &
\end{array}
$$

Moreover, across the contact surface II the pressure and the streamline slope $\tilde{\sigma}$ remain constant, and therefore:

$$
\tilde{\sigma}_{c}=\tilde{\sigma}_{d} \quad P_{c}=P_{d}
$$

To calculate the values of the flowfield variables in regions $c$ and $d$, equations (28) must be integrated. Following the technique presented in reference [2], the integration is performed in an approximate way; therefore, the complete set of equations that it is necessary to solve is:

$$
\begin{aligned}
& R_{5_{a}}=R_{5_{c}} \simeq P_{c}+\left(\frac{\gamma u^{2}}{a^{2} \beta-u^{2} \tilde{\sigma}(\alpha-1)}\right)_{a} \tilde{\sigma}_{c} \\
& R_{2_{a}}=R_{2_{c}} \simeq h_{c}-\left(\frac{a^{2} \omega}{\gamma} \frac{(1+\tilde{\sigma})}{1+\omega \tilde{\sigma}^{2}}\right)_{a} P_{c}+\left(\frac{u^{2} \tilde{\sigma}(1-\omega)}{1+\omega \tilde{\sigma}^{2}}\right)_{a} \tilde{\sigma}_{c} \\
& R_{2_{b}}=R_{2_{d}} \simeq h_{d}-\left(\frac{a^{2} \omega}{\gamma} \frac{(1+\tilde{\sigma})}{1+\omega \tilde{\sigma}^{2}}\right)_{b} P_{d}+\left(\frac{u^{2} \tilde{\sigma}(1-\omega)}{1+\omega \tilde{\sigma}^{2}}\right)_{b} \tilde{\sigma}_{d} \\
& R_{1_{b}}=R_{1_{d}} \simeq P_{d}-\left(\frac{\gamma u^{2}}{a^{2} \beta+u^{2} \tilde{\sigma}(\alpha-1)}\right)_{b} \tilde{\sigma}_{d} \\
& w_{a}=w_{c} \\
& w_{b}=w_{d} \\
& h_{a}^{\circ}=h_{c}^{\circ} \\
& h_{b}^{\circ}=h_{d}^{\circ} \\
& P_{c}=P_{d} \\
& \tilde{\sigma}_{c}=\tilde{\sigma}_{c}
\end{aligned}
$$



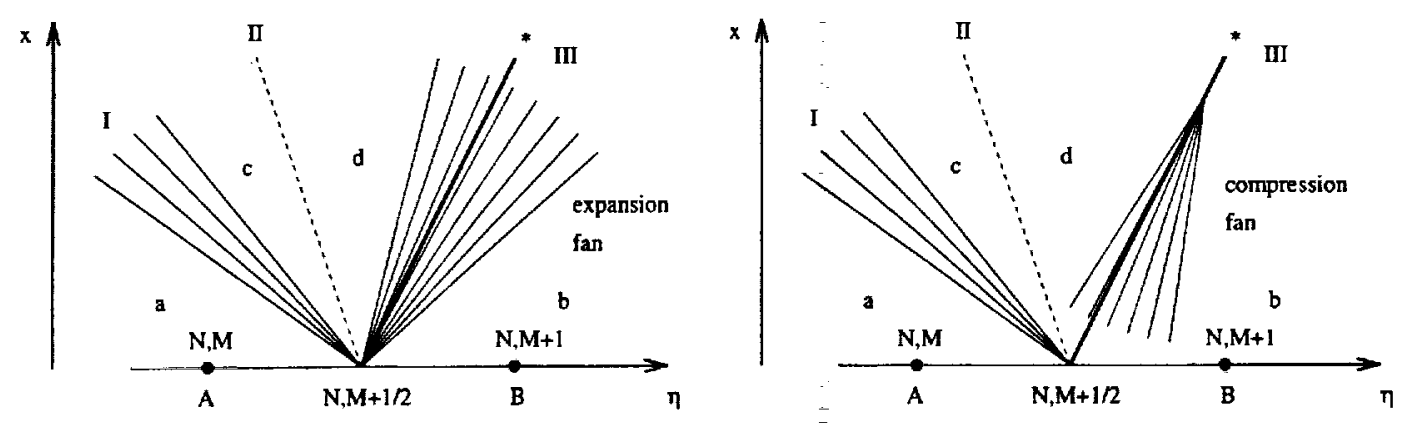

Figure 6: The sonic line

Once regions $c$ and $d$ are known, it is possible to verify if the acoustic waves I and III are expansion or compression fans (that is shock waves). It is also possible to verify in which region the trace of the lateral surface in the $\eta$-x plane is embedded, and therefore to calculate the correct fluxes. In the situation depicted in figure $\overline{\overline{5}}$, for example, the lateral surface is in region $d$, so that the flux across it can be easily found using the values computed there. This also corresponds to splitting the flux difference between $A$ and $B$ in three contributions:

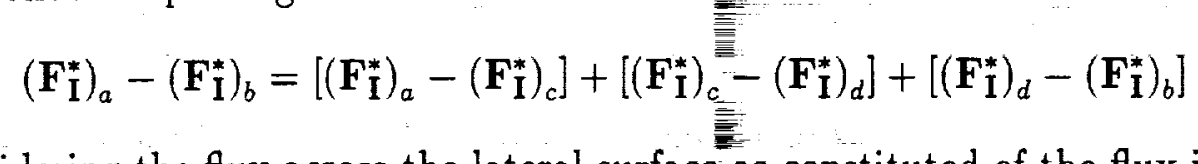

and to considering the flux across the lateral surface as constituted of the flux in $a$ plus the contribution of the left traveling waves:

$$
\left(\mathbf{F}_{\mathbf{I}}^{*}\right)_{N, M+1 / 2}=\left(\mathbf{F}_{\mathbf{I}}^{*}\right)_{a}+\left[\left(\mathbf{F}_{\mathbf{I}}^{*}\right)_{d}-\left(\mathbf{F}_{\mathbf{I}}^{*}\right)_{c}\right] \overline{\overline{\bar{t}}}\left[\left(\mathbf{F}_{\mathbf{I}}^{*}\right)_{c}-\left(\mathbf{F}_{\mathbf{I}}^{*}\right)_{a}\right]=\left(\mathbf{F}_{\mathbf{I}}^{*}\right)_{d}
$$

or of the flux in $b$ plus the contribution of the right traveling waves:

$$
\left(\mathbf{F}_{\mathbf{I}}^{*}\right)_{N, M+1 / 2}=\left(\mathbf{F}_{\mathbf{I}}^{*}\right)_{b}+\left[\left(\mathbf{F}_{\mathbf{I}}^{*}\right)_{d}-\left(\mathbf{F}_{\mathbf{I}}^{*}\right)_{b}\right]=\left(\mathbf{F}_{\mathbf{I}}^{*}\right)_{d}
$$

In case the lateral surface is embedded in an expansion or compression fan, the values of the flowfield variables in correspondence with the 'sonic point' labeled with a star in figure 6 must be known. According to what has been previously stated, the flow here is obtainable from conditions:

$$
\begin{aligned}
& R_{1_{b}} \simeq P^{*}-\left(\frac{\gamma u^{2}}{a^{2} \beta+u^{2} \tilde{\sigma}(\alpha-1)}\right)_{b} \tilde{\sigma}^{\frac{\tilde{\sigma}^{*}}{\bar{m}}} \\
& R_{2_{b}} \simeq h^{*}-\left(\frac{a^{2} \omega}{\gamma} \frac{(1+\tilde{\sigma})}{1+\omega \tilde{\sigma}^{2}}\right)_{b} P^{*}+\left(\frac{u^{2} \tilde{\sigma}(1-\omega)}{1+\omega \tilde{\sigma}^{2}}\right)_{b} \tilde{\sigma}^{*} \\
& u_{b}=w^{*} \\
& h_{b}^{\circ}=h^{*} \\
& \lambda_{5}^{*}=\lambda_{\text {surface }}
\end{aligned}
$$

System (31) can be easily solved through iterations. When the values of the dependent variables in correspondence with the sonic line are known, it is possible to write, in case of an expansion fan:

$$
\left(\mathbf{F}_{\mathbf{I}}^{*}\right)_{N, M+1 / 2}=\left(\mathbf{F}_{\mathbf{I}}^{*}\right)_{b}+\left[\left(\mathbf{F}_{\mathbf{I}}^{*}\right)^{*-}-\left(\mathbf{F}_{\mathbf{I}}^{*}\right)_{b}\right]=\left(\mathbf{F}_{\mathbf{I}}^{*}\right)^{*}
$$


or

$$
\left(\mathbf{F}_{\mathrm{I}}^{*}\right)_{N, M+1 / 2}=\left(\mathbf{F}_{\mathbf{I}}^{*}\right)_{a}+\left[\left(\mathbf{F}_{\mathbf{I}}^{*}\right)^{*}-\left(\mathbf{F}_{\mathrm{I}}^{*}\right)_{a}\right]=\left(\mathbf{F}_{\mathbf{I}}^{*}\right)^{*}
$$

and in case of a compression fan:

$$
\left(\mathbf{F}_{\mathrm{I}}^{*}\right)_{N, M+1 / 2}=\left(\mathbf{F}_{\mathbf{I}}^{*}\right)_{b}+\left[\left(\mathbf{F}_{\mathbf{I}}^{*}\right)_{d}-\left(\mathbf{F}_{\mathbf{I}}^{*}\right)^{*}\right]
$$

or

$$
\left(\mathbf{F}_{\mathrm{I}}^{*}\right)_{N, M+1 / 2}=\left(\mathbf{F}_{\mathrm{I}}^{*}\right)_{a}+\left[\left(\mathbf{F}_{\mathrm{I}}^{*}\right)_{b}-\left(\mathbf{F}_{\mathrm{I}}^{*}\right)^{*}\right]+\left[\left(\mathbf{F}_{\mathbf{I}}^{*}\right)_{d}-\left(\mathbf{F}_{\mathrm{I}}^{*}\right)_{a}\right]=\left(\mathbf{F}_{\mathrm{I}}^{*}\right)_{b}+\left(\mathbf{F}_{\mathrm{I}}^{*}\right)_{d}-\left(\mathbf{F}_{\mathbf{I}}^{*}\right)^{*}
$$

The approximation that the acoustic waves are isentropic also in case they are shocks could lead to some inaccuracies when strong shock waves are concerned. For this reason, when the presence of a strong compression is recognized through an acoustic wave, an exact Riemann problem solver is switched on. In the case of figure 6 , this consists in imposing the Rankine-Hugoniot conditions across wave III, the Prandtl-Meyer conditions across wave I and the constancy of $p$ and $\tilde{\sigma}$ across wave II.

\subsection{Evaluation of the lateral diffusive fluxes}

To evaluate the diffusive fluxes contained in vector $\mathbf{F}_{\mathbf{V}}^{*}$ it is necessary to compute $u, v$ and $w$ and the gradients of $u, v, w$ and $T$ in the $y-z$ plane in correspondence with the trace of the considered lateral surface.

Gradients are calculated using the Gauss' theorem:

$$
\int_{\mathcal{V}} \nabla f d V=\int_{\mathcal{S}} f \cdot \vec{n} d S
$$

and applying it to the $y-z$ plane containing surfaces labeled with 4 . The discretized form of equation (32) reduced to two dimensions is:

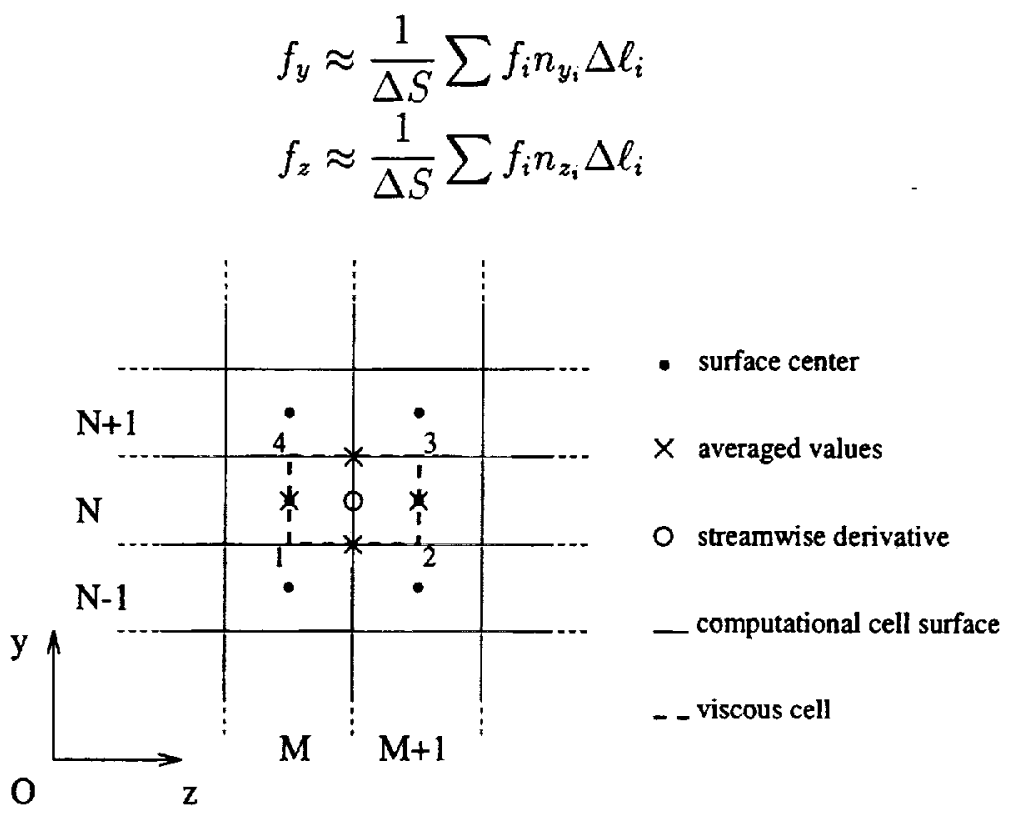

Figure 7: The viscous cell 
where $\Delta \ell_{i}$ indicates each side of the viscous cell that has to be built up around the trace of the considered lateral surface.

The values of $f_{i}$ are computed as the averaged value of $f$ itself in that point. With reference to figure 7 , we have for instance:

$$
\begin{aligned}
& \left(f_{y}\right)_{N, M+1 / 2}=\frac{1}{S_{1234}}\left(f_{12} \Delta \ell_{12_{y}}+f_{23} \Delta \ell_{23_{y}}+f_{34} \Delta \ell_{34_{y}}+f_{41} \Delta \ell_{41 y}\right) \\
& \left(f_{z}\right)_{N, M+1 / 2}=\frac{1}{S_{1234}}\left(f_{12} \Delta \ell_{12 z}+f_{23} \Delta \ell_{23_{z}}+f_{34} \Delta \ell_{34_{z}}+f_{41} \Delta \ell_{41_{z}}\right)
\end{aligned}
$$

where

$$
\begin{aligned}
& f_{12}=\frac{1}{4}\left(f_{N-1, M}+f_{N-1, M+1}+f_{N, M+1}+f_{N, M}\right) \\
& f_{23}=f_{N, M+1} \\
& f_{34}=\frac{1}{4}\left(f_{N, M}+f_{N, M+1}+f_{N+1, M+1}+f_{N+1, M}\right) \\
& f_{41}=f_{N, M}
\end{aligned}
$$

On the other hand, the values of $u, v$ and $w$ in correspondence with the trace of the lateral surface are simply computed as the averages of the values in the adjacent cells:

$$
f_{N, M+1 / 2}=\frac{f_{N, M}+f_{N, M+1}}{2}
$$

\subsection{Boundary conditions}

Boundary conditions have been implemented imposing no slip and adiabatic or isothermal conditions at the wall and, for what concerns the pressure gradient, reducing Navier-Stokes equations to the boundary layer limit:

$$
\begin{aligned}
& q_{w}=0 \\
& \begin{cases}(\nabla T)_{w}=0 & \text { for adiabatic wall } \\
T_{w} \text { imposed } & \text { for isothermal wall }\end{cases} \\
& (\nabla p)_{w}=0
\end{aligned}
$$

\subsection{Initial conditions}

The use of a space marching technique requires the specification of the flow conditions across the inlet surface. The geometrical configurations considered here posses a leading edge sufficiently thin to produce a shock wave attached to the body, permitting us to start the integration directly from the leading edge, where freestream conditions are imposed. It is necessary, however, to start with short integration steps, to allow the viscous layer to establish as quickly as possible. 


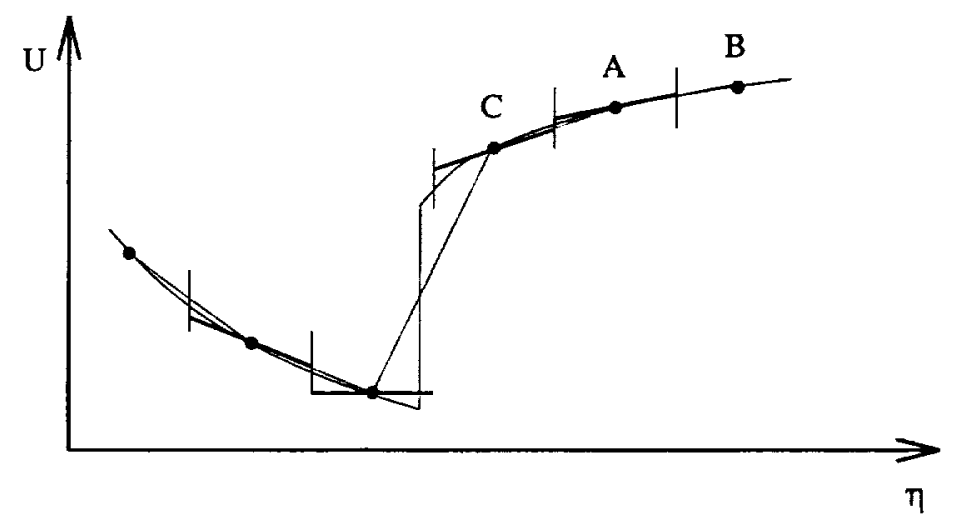

Figure 8: The ENO scheme

\subsection{Second order accuracy}

Second order accuracy is achieved following the guidelines of the Essentially Non Oscillatory schemes for shock capturing techniques, originally proposed for time-dependent problems [4]. In the present case, the initial data distribution of the primitive variables $\mathbf{U}=\{p, u, v, w, h\}^{T}$ along the $\eta$ direction is considered to be piecewise linear. With reference to figure 8 , it is for instance:

$$
\begin{aligned}
& \mathbf{U}=\mathbf{U}_{A}+\mathbf{s}_{A}\left(\eta-\eta_{A}\right) \quad \text { in } \mathrm{A} \\
& \mathbf{U}=\mathbf{U}_{B}+\mathbf{s}_{B}\left(\eta-\eta_{B}\right) \quad \text { in } \mathrm{B}
\end{aligned}
$$

The slopes $\mathbf{s}$ are chosen in the ENO fashion to avoid spurious oscillations at flow discontinuities, that is, for each element $s_{A}$ of vector $\mathbf{s}_{\mathbf{A}}$ :

$$
s_{A}=\operatorname{minmod}\left(s_{A}^{+}, s_{A}^{-}\right)=\left\{\begin{array}{lll}
\min \left(\left|s_{A}^{+}\right|,\left|s_{A}^{-}\right|\right) & \text {if } & s_{A}^{+} \cdot s_{A}^{-}>0 \\
0 & \text { if } & s_{A}^{+} \cdot s_{A}^{-}<0
\end{array}\right.
$$

with

$$
\begin{aligned}
& s_{A}^{+} \simeq \frac{U_{B}-U_{A}}{d(B, A)} \\
& s_{A}^{-} \simeq \frac{U_{A}-U_{C}}{d(A, C)}
\end{aligned}
$$

The discontinuity originating the Riemann problem is thus defined by the values:

$$
\begin{aligned}
& \left(\mathrm{U}_{N, M+1 / 2}\right)_{L}^{\prime} \simeq \mathrm{U}_{A}+\mathrm{s}_{A} \frac{\eta_{B}-\eta_{A}}{2} \simeq \mathrm{U}_{A}+\mathrm{s}_{A} \frac{d(A, B)}{2} \\
& \left(\mathrm{U}_{N, N+1 / 2}\right)_{R}^{\prime} \simeq \mathrm{U}_{B}-\mathrm{s}_{A} \frac{\eta_{B}-\eta_{A}}{2} \simeq \mathrm{U}_{B}-\mathrm{s}_{A} \frac{d(A, B)}{2}
\end{aligned}
$$

Second order accuracy in the hyperbolic space marching direction $x$ is obtained by using the (complete) quasilinear form of the governing equations, which makes it possible to obtain the derivatives $p_{x}, u_{x}, v_{x}, w_{x}$ and $h_{x}$. The necessary derivatives in the $y$ and $z$ direction are evaluated using the Gauss' theorem as in the computation of the viscous fluxes. Therefore, the final values for defining the Riemann problem are provided by:

$$
\begin{aligned}
& \left(\mathbf{U}_{N, M+1 / 2}\right)_{L}=\mathbf{U}_{A}+\mathbf{s}_{A} \frac{d(A, B)}{2}+\left(\mathbf{U}_{x}\right)_{A} \frac{\Delta x}{2} \\
& \left(\mathbf{U}_{N, M+1 / 2}\right)_{R}=\mathbf{U}_{B}-\mathbf{s}_{B} \frac{d(A, B)}{2}+\left(\mathbf{U}_{x}\right)_{B} \frac{\Delta x}{2}
\end{aligned}
$$




\subsection{Evaluation of the integration step size}

The amplitude of the integration step is chosen according to the inviscid CFL condition modified with a viscous correction. The adopted procedure is the following:

$$
\Delta x=\min \left(\Delta x_{y}, \Delta x_{z}\right)
$$

with

$$
\begin{aligned}
\Delta x_{y} & =K_{v i s} \frac{\Delta y}{\left|\lambda_{y}\right|_{\max }} \\
\Delta x_{z} & =K_{v i s} \frac{\Delta z}{\left|\lambda_{z}\right|_{\max }} \\
K_{v i s} & =\frac{1}{1+\frac{2 \mu}{\operatorname{Re} \rho u \min (\Delta y, \Delta z)}}
\end{aligned}
$$

\section{Results and Conclusions}

To verify the capacity of the above discussed methodology to solve complex flowfields, a numerical study of symmetric supersonic corner flows in laminar regime is presented. The obtained results are also compared with experimental data taken from the literature, in particular from reference [8].

To match with experiments, the following geometrical and fluid-dynamic conditions have been considered:

\begin{tabular}{|c|c|c|c|c|c|c|c|}
\hline$\delta_{1}$ & $\delta_{2}$ & $\theta$ & $M_{\infty}$ & $R e_{\infty} / m$ & $\operatorname{Pr}$ & $T_{\infty}[\mathrm{K}]$ & $T_{w}[\mathrm{~K}]$ \\
\hline $8^{\circ}$ & $8^{\circ}$ & $90^{\circ}$ & 12.3 & $5 \times 10^{6}$ & 0.72 & 45.3 & 300 \\
\hline
\end{tabular}

where the meaning of $\delta$ and $\theta$ is shown in figure 9 .

The fluid-dynamic pattern typical of this kind of flows consists of a system of five shock waves. Two of them, which separate regions I and II in figure 10, are generated by the presence of the wedges; the remaining three are due to the presence of a Mach disk (irregular reflection) in the region where the previous two converge. Contact surfaces directed towards the symmetry plane are generated at points where shocks interact (triple points) because of the different levels of entropy produced by the wave system on either side of the interaction. Shock waves separating regions II and IV impinge on the boundary layer and are reflected as expansion waves, which in turn encounter the slip surfaces and are transformed in compression waves. The interaction between the impinging shock and the boundary layer provokes the scparation of the latter in the crosswise direction. Therefore, a streamwise vortex develops. resulting as an obstacle to the crossflow, and thus generating a compression analogous to the one typical of two dimensional supersonic flows over a ramp. 
In the following, numerical results obtained using a stretched computational grid composed of 100x100 cells will be shown. The stretching function used is:

$$
\xi_{N}=\xi_{w}+\left(\xi_{\max }-\xi_{w}\right)\left[1-\beta_{\xi}+\frac{2 \beta_{\xi}}{1+\left(\frac{\beta_{\xi}+1}{\beta_{\xi}-1}\right)^{\psi}}\right]
$$

with

$$
\psi=\left(\frac{N_{\xi_{\max }}-N}{N_{\xi_{\max }}-1}\right)
$$

In particular, a stretching parameter $\beta_{y}=\beta_{z}=1.03$ has been used.

The external boundaries have been defined starting with $y_{\max }=0.055$ and $z_{\max }=0.055$ at the inlet surface; proceeding with the integration, the upper and lateral boundary surfaces have been kept parallel to the opposite walls.

The presence of the vortical structures and of the related compression fan transports the three-dimensional effects due to the corner at noticeable distances from it inside the shock layer, making it necessary to fix rather ample external boundaries. If a square or rectangular grid is used, this results in a lot of points outside the shock layer. To avoid wasting time in computing points for which it is known a priori that the freestream conditions apply, a procedure has been implemented that detects the position of the shock layer at each step of integration and solves the flowfield only in the part of the domain containing it.

In figure $11 \mathrm{a}$, crossflow streamlines corresponding to $\mathrm{x}=0.09 \mathrm{~m}$ are plotted. The presence of a large streamwise vortex split in two parts that are tied by a saddle point can be clearly seen. Below it, close to the wall, a small secondary vortex is captured thanks to the great refinement of the mesh. This picture is enlarged in figure 11c. In figure $11 \mathrm{~b}$, pressure contours are presented using the same scale. The patterns described by the sketch of figure 10 are found.

In figure 12 , limiting streamlines at the wall are plotted; crossflow streamlines have been placed beside for a better comprehension of the picture. The separation and reattachment of the primary double vortex are well evidenced, and also the presence of the secondary vortex at the wall can be perceived.

The same computed results presented above are now compared with experimental data extracted from reference [8]. In figure 13, pitot pressure contours corresponding to a distance from the leading edge of $0.09 \mathrm{~m}$ are shown. The contours show a qualitative good agreement. In fact, the wedge-shock position is not exactly the same, as the computed one corresponds to a local slope of about $4.9^{\circ}$ with respect to the wedge, versus an angle of about $5.5^{\circ}$ for the experiments (the inviscid value should be $3.3^{\circ}$ ). Nevertheless, the maximum pitot pressure value is 6.70 for the computation, and experiments show their highest value on the contour corresponding to 6.42 .

In addition, in figure $14 \mathrm{a}$, numerical and experimental static pressure at the wall are overlayed, showing a satisfactory agreement. In figure $14 \mathrm{~b}$, the flow direction at the wall is compared. The two curves are very similar close to the corner, as demonstrated by the fact that the reattachment of the primary vortex is detected in the same position. Nevertheless, away from the intersection of the ramps, some not negligible differences start to appear. In 
the computation, the secondary vortex is smaller and closer to the corner (though not to such a great extent) and the separation of the primary vortex is located in a different position with respect to the experiments. Such discrepancies might possibly be due to the fact that, approaching the lateral boundaries of the model, experiments are affected by side-effects or by interference with the tunnel boundary layer. Finally, in figure 14c, the heat flux at the wall is compared. As it could be expected after the previous discussion, the results are similar as far as the reattachment of the primary vortex is concerned, with the peak of heat transfer in the same position and showing a similar magnitude. Conversely, the location of the peak related to the secondary vortex is different, though the value is the same. Other discrepancies concern the fact that, in the numerical results, we find no trace of the peak which, in the experimental curve, is signaled at $\bar{y} \simeq 0.09$; in effect, it seems difficult to justify its presence, since no visible reattachment is detected in that position, neither in the experiment. Last, it can be noticed that the computation predicts an almost null heat flux locally at the corner, in contrast with the experiments; in this case we think it is reasonable to trust the numerical result, since very close to the corner the temperature varies very smoothly, and on the other hand the measurement technique used cannot approach close to the corner.

In order to adequately address the accuracy of the computation, the results obtained with the $100 \times 100$ grid are compared with those resulting from a $60 \times 60$ grid. The main features of the flowfield are unchanged, as can be seen from figure 15, where pitot pressure contours are shown. The major difference is related to the secondary vortex: with the finer mesh it is fully captured, while with the coarser one its presence is just sensed. A proof to this statement can be found in figures 16 and 17. In figure 16, crossflow streamlines are presented: in the left picture (60x60 mesh), the undulation of the crossflow streamlines suggests the presence of the secondary vortex, but in the right one $(100 \times 100 \mathrm{mesh})$, the vortex is evident. In figure 17 , the flow direction at the wall is shown again: it can be seen that in the case of the finer mesh $(100 \times 100)$, a crossflow reversal is present at about $\bar{y}=0.12$, while with the coarser mesh $(60 \times 60)$ this feature does not exist.

In conclusion, the agreement arising from the comparison appears to be satisfactory, though some minor discrepancies appear.

Therefore, the numerical method proposed seems to be promising, as it permits one to investigate complex three-dimensional flowfields in great detail of a reasonable computational cost.

\section{References}

[1] Y. C. Vigneron, J. V. Rakich, and J. C. Tannehill, "Calculation of supersonic viscous flow over delta wings with sharp subsonic leading edges," Technical Memorandum 78500, NASA, June 1978.

[2] M. Pandolf, "Computation of steady supersonic flows by a flux difference splitting method," Journal of Computers and Fluids, vol. 10, no. 1, 1985.

[3] R. Marsilio, "Vortical solutions in supersonic corner flows," AIAA Journal, vol. 31, September 1993. 
[4] A. Harten, B. Engquist, S. Osher, and S. R. Chakravarthy, "Uniformly high order accurate Essentially Non-Oscillatory schemes, III," Journal of Computational Physics, vol. 71, 1987.

[5] D. A. Anderson, J. C. Tannehill, and R. H. Pletcher, Computational Fluid Mechanics and Heat Transfer, ch. 8. New York: McGraw-Hill Book Company, 1984.

[6] J. J. Korte, "An explicit upwind algorithm for solving the Parabolized Navier-Stokes equations," Technical Paper 3050, NASA, February 1991.

[7] J. H. Morrison and J. J. Korte, "Implementation of Vigneron's streamwise pressure gradient approximation in the PNS equations," in AIAA 30th Aerospace Sciences Meeting and Exhibit, (Reno, Nevada), Jan. 6-9 1992. AIAA Paper 92-0189.

[8] D. Hummel, "Experimental investigations on blunt bodies and corner configurations in hypersonic flow," in Aerodynamics of hypersonic lifting vehicles, 1987. AGARD-CP-428, paper 6. 


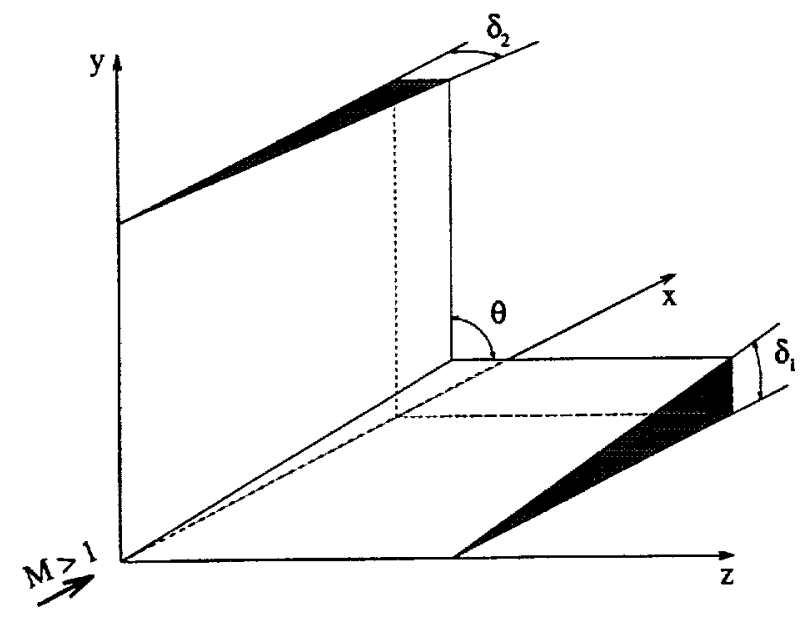

Figure 9: Test configurations

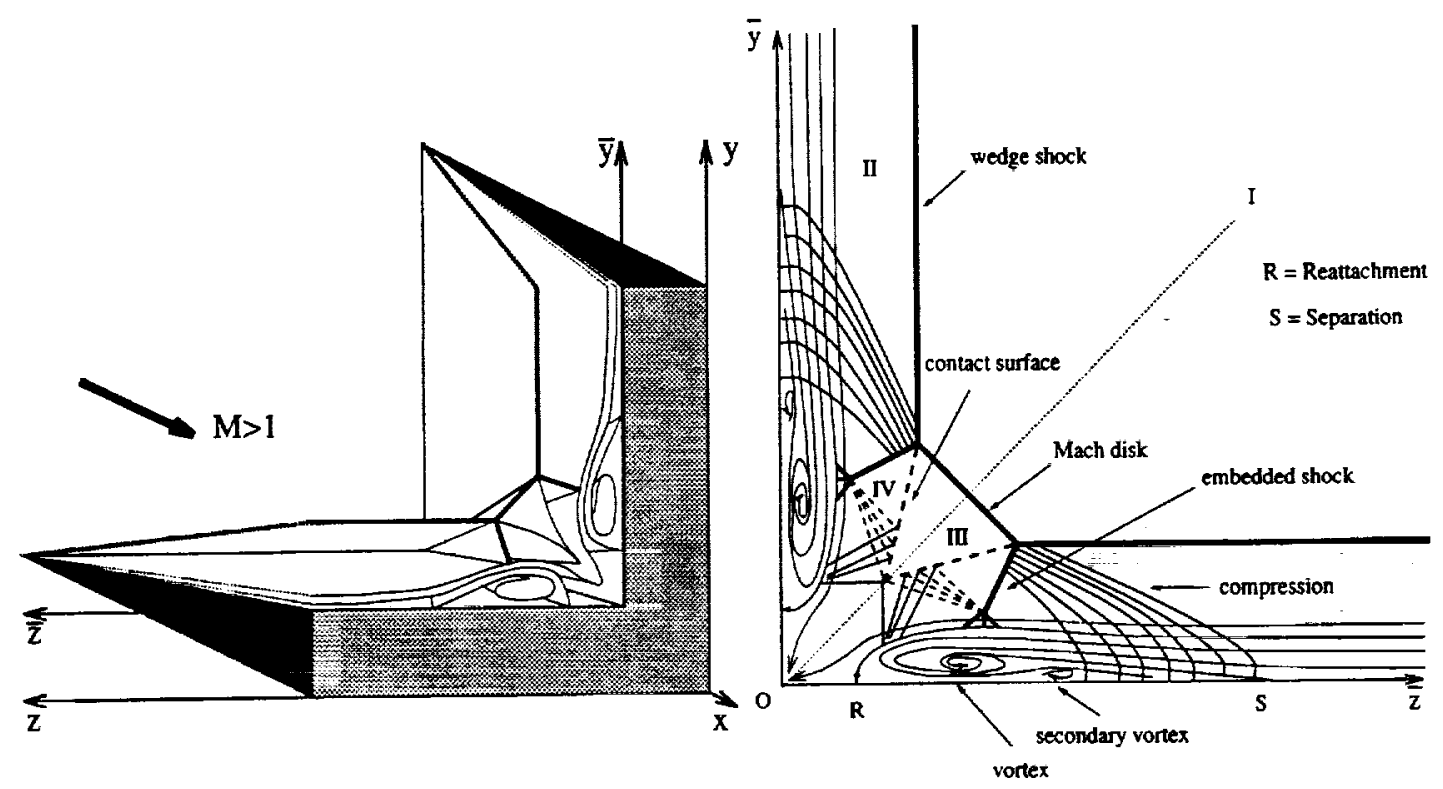

Figure 10: Flowfield configuration 

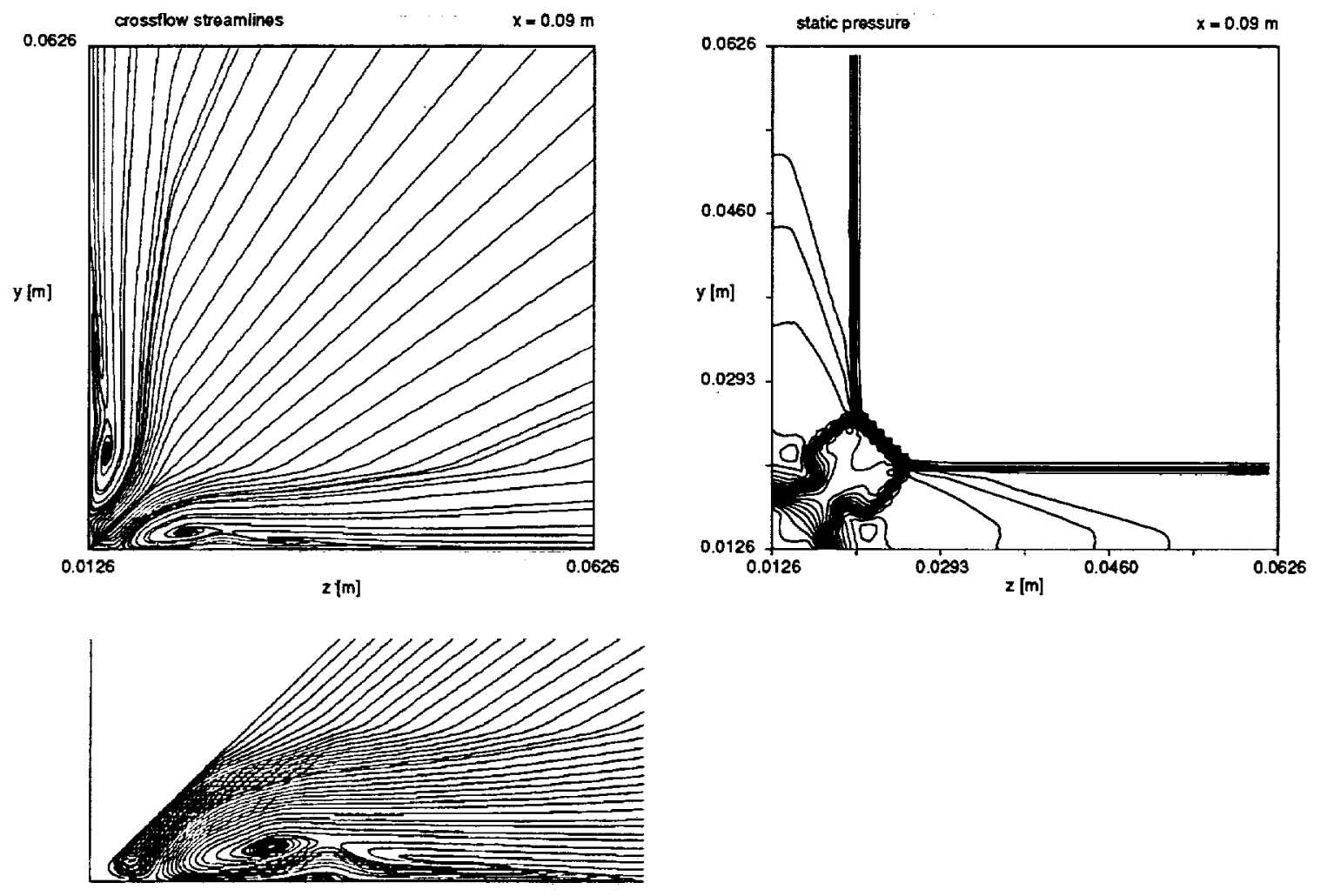

Figure 11: Computed crossflow streamlines and static pressure contours.
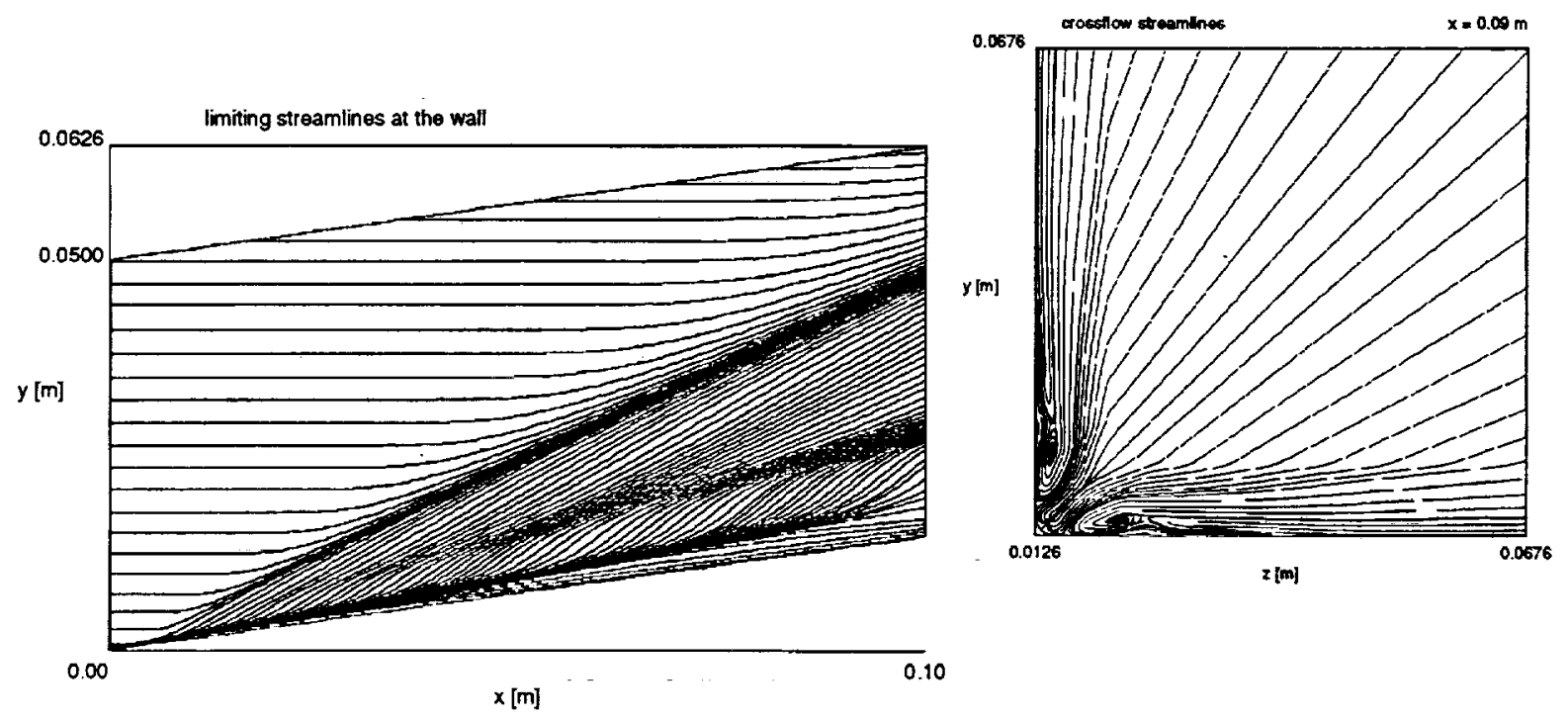

Figure 12: Limiting streamlines at the wall and crossflow streamlines. 
a)
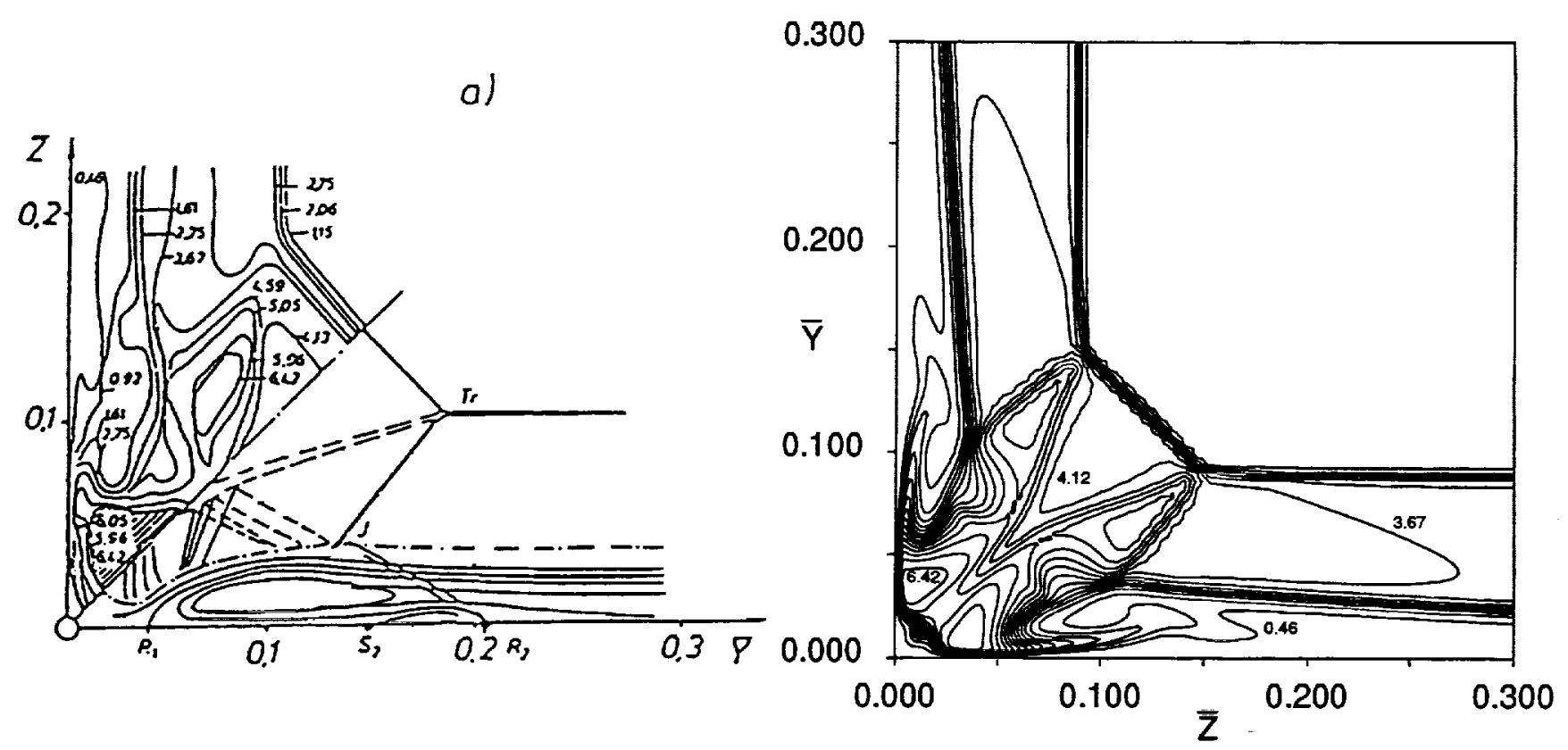

Figure 13: Pitot pressure contours: (a)experiment, (b)computation.

Po is the pressure for the iaricid $2 D$ wedge

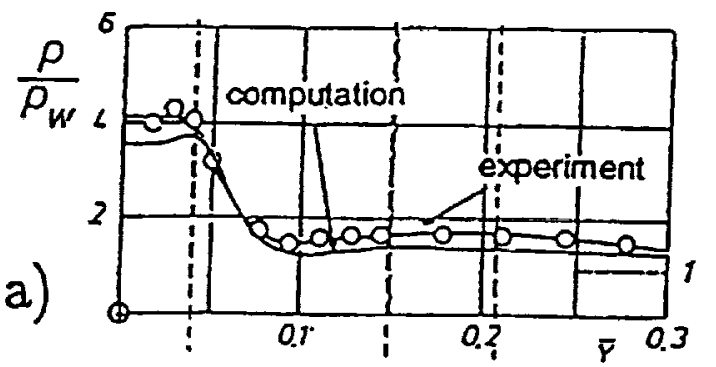

Qu is the heat Aux at the wall for the 2D wedge

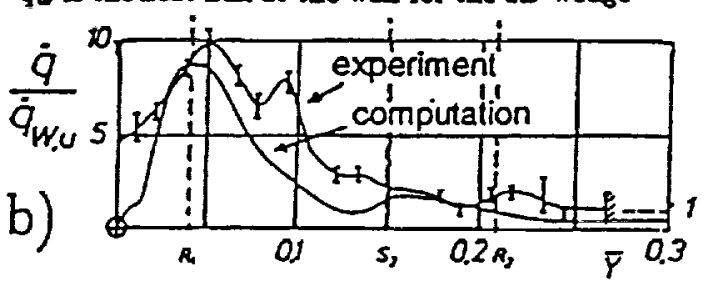

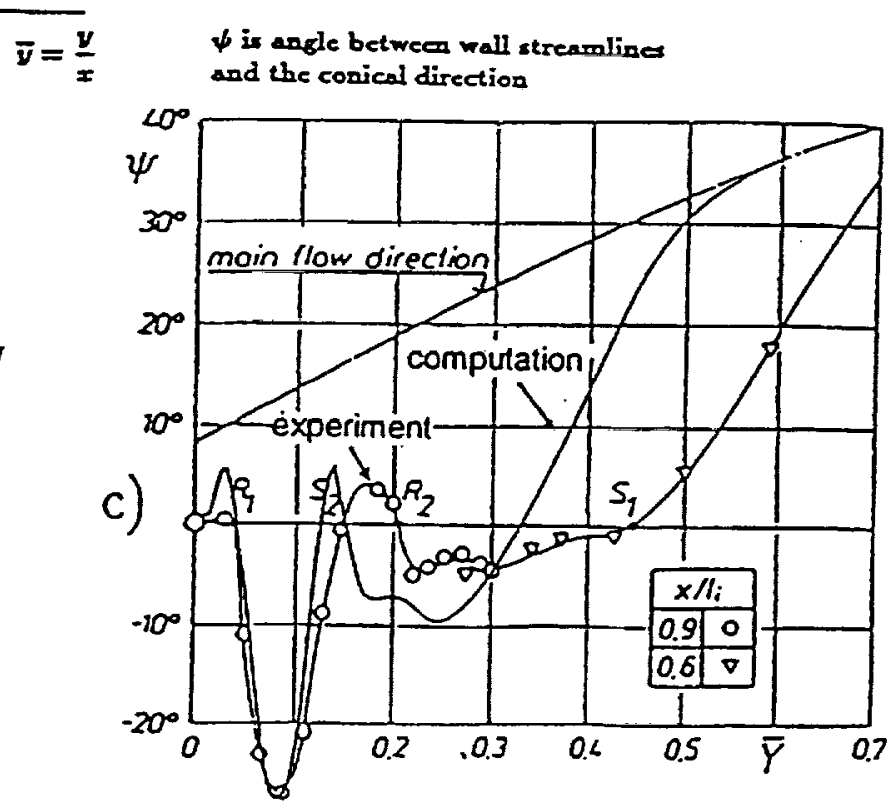

Figure 14: Wall pressure (a), flow direction (b) and heat fluxes (c) at the wall: experiment and computation. 

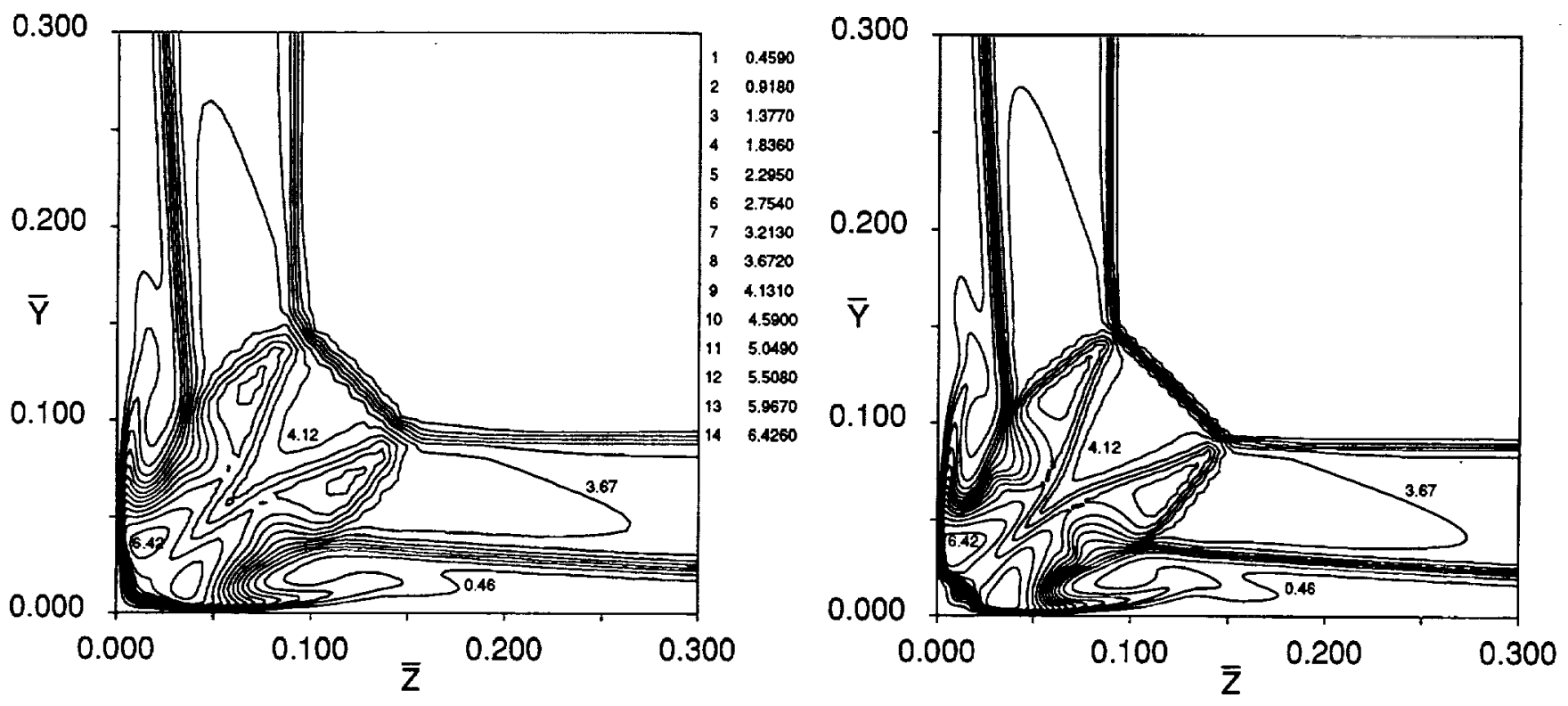

Figure 15: Pitot pressure contours computed with different meshes: (a)60x60 mesh, (b) $100 \times 100$ mesh.
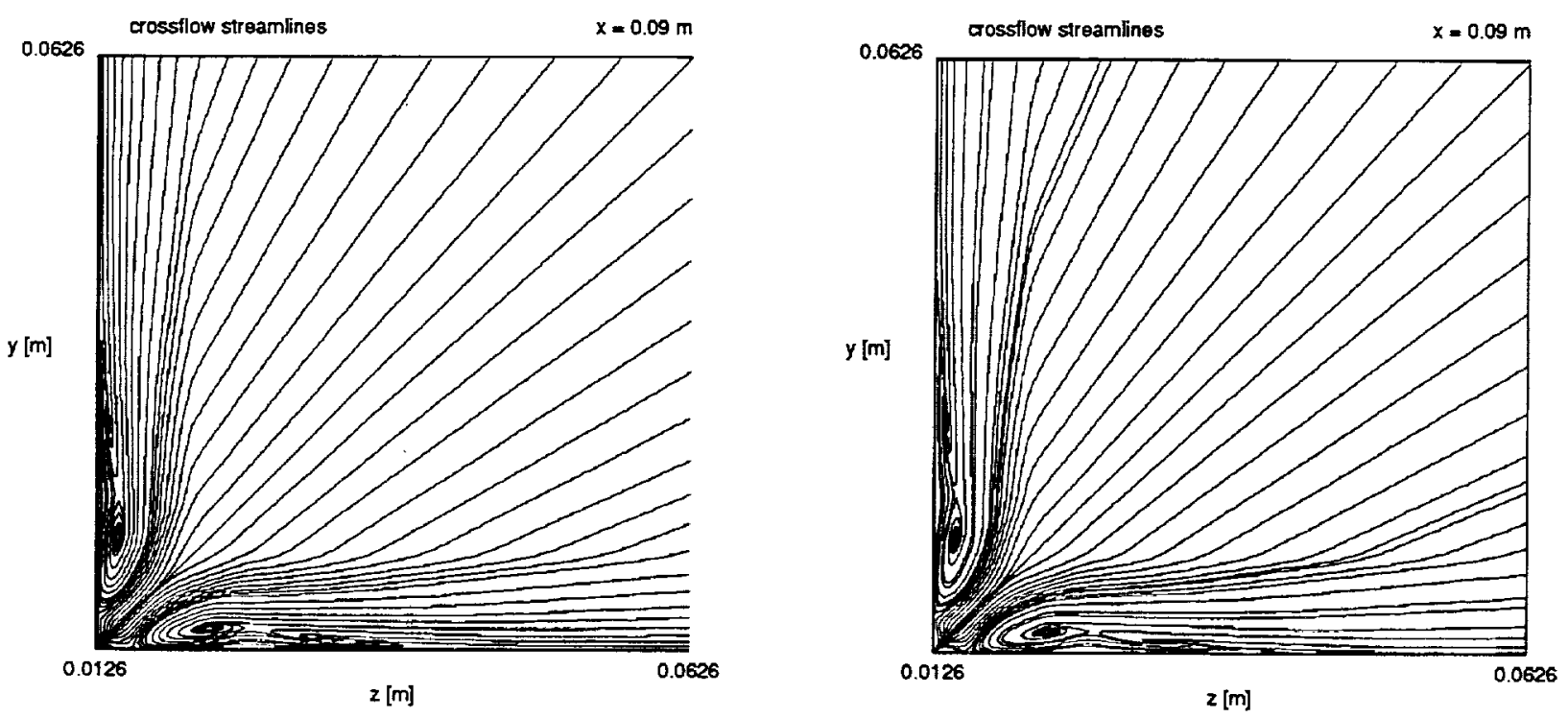

Figure 16: Computed crossflow streamlines: (a)60x60 mesh, (b)100x100 mesh. 


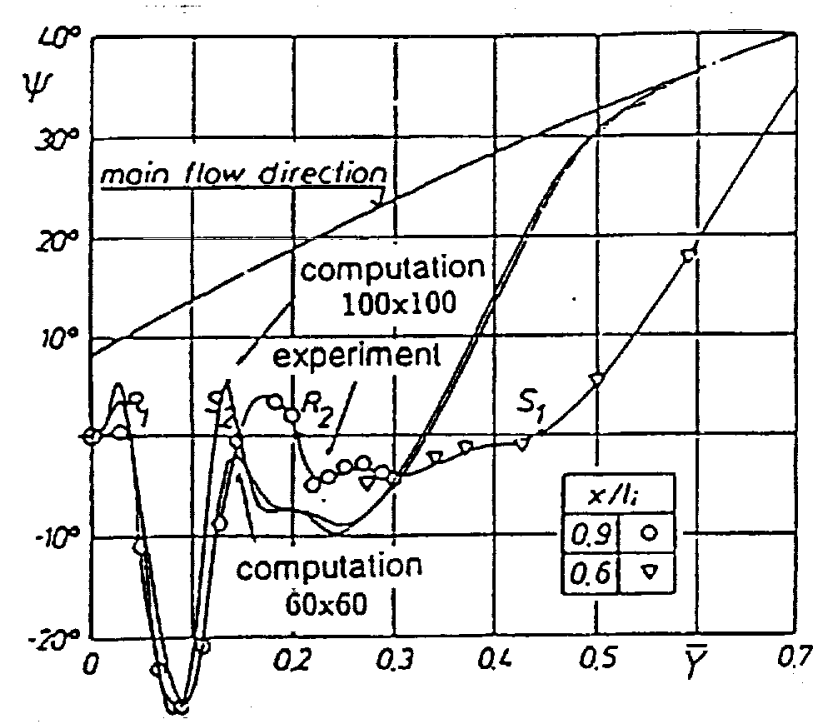

Figure 17: Flow direction at the wall: (a)60x60 mesh, (b)100x100 mesh. 


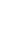




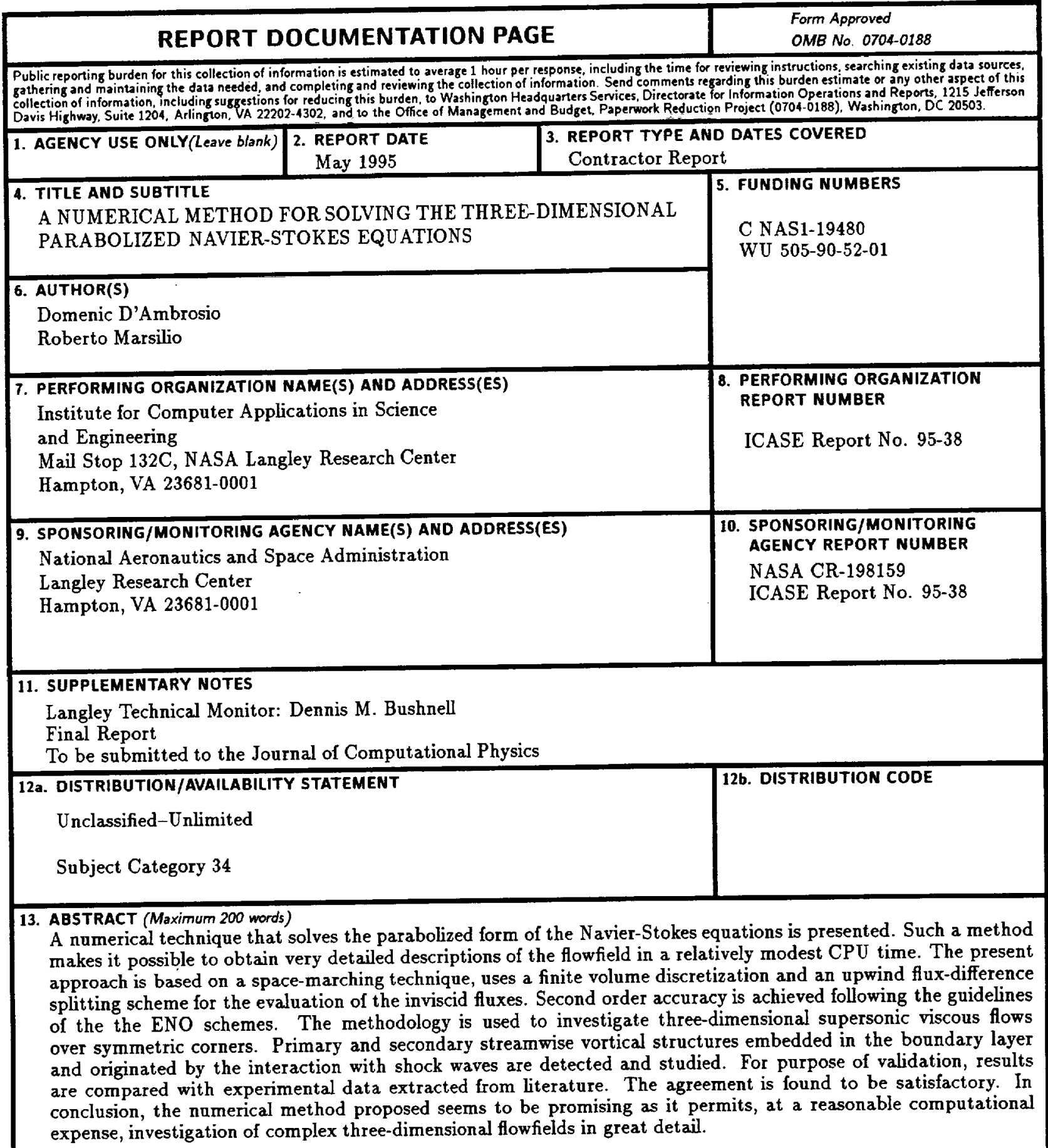



. 